\title{
DERECHOS LABORARLES DE LAS Y LOS JÓVENES INDÍGENAS EN EL MARCO DE LA EXPERIENCIA MIGRATORIA A LAS CIUDADES EN MÉXICO
}

\author{
LABOR RIGHTS OF INDIGENOUS YOUTH IN THE FRAMEWORK OF THE \\ MIGRATION EXPERIENCE TO CITIES IN MEXICO
}

\author{
DIREITOS TRABALHISTAS DA JUVENTUDE INDÍGENA NO CONTEXTO \\ DA EXPERIÊNCIA DE MIGRAÇÃO PARA CIDADES NO MÉXICO
}

\section{Resumen}

La pretensión que guarda este artículo es colocar la discusión de los derechos laborales de las y los jóvenes indígenas en los contextos de migración, vinculando un debate político con los hallazgos que diferentes investigaciones han encontrado al estudiar la participación de estos actores en el fenómeno migratorio a las ciudades. La base de información que presentaré proviene, por un lado, del estudio que he realizado en los últimos años sobre las condiciones estructurales y subjetivas de mujeres jóvenes indígenas migrantes en la zona metropolitana del valle de México y mujeres indígenas organizadas; por otro, de una la recopilación bibliográfica y documental sobre jóvenes indígenas en México. Se concluye que, el ejercicio de los derechos laborales para las juventudes indígenas va más allá de programas de formación, capacitación y participación en el sistema educativo, o de las condiciones que haya en los mercados laborales, ha considerarse al mismo tiempo, el apoyo a proyectos productivos que involucre a las generaciones más jóvenes en los propios lugares de origen, una revisión de cómo afectan los megaproyectos las condiciones de vida y laborales de los jóvenes presentes y de las generaciones futuras, así mismo, es importante considerar la experiencia y la condición juvenil como valiosa en sí misma, pero entendiéndola siempre en un amplio contexto de relaciones sociales de poder.

Palabras clave: derechos laborales, jóvenes indígenas, migración, ciudad. 


\begin{abstract}
The aim of this article is to place the discussion of the labor rights of indigenous youth in the contexts of migration, linking a political debate with the findings that different investigations have found when studying the participation of these actors in the migration phenomenon. the cities. The information base that I will present comes, on the one hand, from the study I have done in recent years on the structural and subjective conditions of young indigenous migrant women in the metropolitan area of the Valley of Mexico and organized indigenous women; on the other, from a bibliographic and documentary collection of indigenous youth in Mexico. It is concluded that, the exercise of labor rights for indigenous youth goes beyond training programs, training and participation in the educational system, or the conditions that exist in labor markets, at the same time, support for productive projects that involve younger generations in their own places of origin, a review of how megaprojects affect the living and working conditions of young people present and of future generations, likewise, it is important to consider the experience and condition juvenile as valuable in itself, but always understanding it in a broad context of social relations of power.
\end{abstract}

Keywords: labor rights, indigenous youth, migration, city.

\title{
Resumo
}

O objetivo deste artigo é colocar a discussão dos direitos trabalhistas da juventude indígena nos contextos de migração, articulando um debate político com os achados que diferentes investigações encontraram ao estudar a participação desses atores no fenômeno migratório. as cidades. A base de informações que apresentarei vem, por um lado, do estudo que fiz nos últimos anos sobre as condições estruturais e subjetivas de jovens mulheres migrantes indígenas na região metropolitana do Vale do México e mulheres indígenas organizadas; do outro, de uma coleção bibliográfica e documental de jovens indígenas no México. Conclui-se que, o exercício dos direitos trabalhistas para a juventude indígena vai além dos programas de treinamento, treinamento e participação no sistema educacional, ou das condições que existem nos mercados de trabalho, ao mesmo tempo, projetos produtivos que envolvam as gerações mais jovens em seus próprios locais de origem, uma revisão de como os megaprojetos afetam as condições de vida e de trabalho dos jovens presentes e das futuras gerações, da mesma forma, é importante considerar a experiência e condição juvenil como valioso em si mesmo, mas sempre entendendo-o num contexto amplo de relações sociais de poder.

Palavras-chave: direitos trabalhistas, juventude indígena, migração, cidade. 


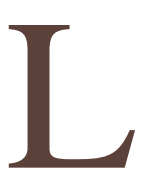

a migración constituye en las últimas décadas una experiencia fundamental para la mayoría de las personas consideradas indígenas. Si bien "el patrón migratorio predominante sigue siendo ruralurbano, y el perfil demográfico de los migrantes es en su mayoría masculino y en edades productivas" (GRANADOS y QUEZADA, 2018:327), las mujeres y los jóvenes -mujeres y varones- han contribuido a engrosar en las últimas tres décadas los flujos de desplazamiento a las ciudades de las personas indígenas.

Específicamente, la migración para las y los jóvenes se ha vuelto central en su experiencia porque alguien de su familia ha debido migrar o porque ellos mismos son protagonistas de los desplazamientos.

La migración, no obstante, es un fenómeno heterogéneo en razón de las características de los individuos y de las condiciones en las que se realizan, de las particularidades de los lugares de salida y de llegada, así como de los motivos que la inducen.

Entre los porqués de migrar para las y los jóvenes indígenas, encontramos una constante: lo hacen en la búsqueda de oportunidades para la sobrevivencia y reproducción de sí mismos, sus familias, comunidades y pueblos, es decir, migran para trabajar. Al respecto la Comisión para el Desarrollo de los Pueblos Indígenas (2015: s/p) señala que, "La condición de actividad o la inserción de la fuerza de trabajo en el mercado laboral muestra la estrecha vinculación entre la actividad económica de la población indígena y su condición migratoria".

En el presente artículo me interesa reflexionar cómo se concatena la etnicidad indígena con la edad, el género y la clase en la forma en la que se insertan las y los jóvenes indígenas en los mercados laborales que les ofrece la ciudad. Con base en diferentes cartas de derechos me interesa señalar hasta qué punto este grupo social tiene la posibilidad de ejercer sus derechos laborales en la migración a la ciudad.

Las reflexiones que presento a lo largo de este artículo provienen en su mayor parte de la información recabada en el estudio de la experiencia juvenil en el marco de las condiciones estructurales y subjetivas de mujeres jóvenes indígenas migrantes en la zona metropolitana del valle de México (LÓPEZ, 2017) y recientemente, de la investigación colaborativa que llevó a cabo con mujeres indígenas organizadas (LÓPEZ, 2018), en la que nos hemos dado la tarea de explorar cuáles son las condiciones que permiten a las jóvenes indígenas contemporáneas conformarse como un sujeto de derecho en los ámbitos familiares, comunitarios y dentro de las 
políticas públicas en la escala local, nacional e internacional.

El artículo se compone de tres partes; en la primera se presenta una breve contextualización de la relación que tienen las y los jóvenes indígenas con el trabajo para después presentar en un segundo apartado, una síntesis de los derechos laborales expresados en diferentes instrumentos en relación con la población indígena. Finalmente, expongo algunas experiencias que tienen las y los jóvenes indígenas que migran a la ciudad para trabajar, a partir de las cuales intentaré mostrar las formas de inserción laboral de acuerdo a cómo se sintetiza la etnicidad con la edad, el género y la clase social, proponiendo que si bien el ejercicio o violación de derechos laborales para las personas indígenas está marcada por su identidad étnica, cobra matices que es necesario advertir como un horizonte heterogéneo y de diversidad en el contexto de la desigualdad estructural que aqueja a la población indígena y a los jóvenes que forman parte de ella.

\section{El trabajo como espacio de socialización para las y los jóvenes indígenas}

La juventud indígena está inmersa dentro la existente red de relaciones sociales. Por lo tanto, los jóvenes no pueden ser entendidos al margen de la sociedad en la que están insertos en relaciones e interacciones que les distinguen de otros grupos de edad por ciertas normas, comportamientos, sentidos y ritos particulares, entre ellos destaca una posición muy específica que la niñez y las juventudes tienen en las sociedades indígenas, pues partimos de observar que desde edades muy tempranas: "La socialización de los niños y las niñas indígenas se vincula con la incorporación al sistema productivo familiar y comunal" (LÓPEZ, 2004, en Del Popolo et al 2009: 38), por lo que: “ser joven en una comunidad indígena significa asumir roles $y$ funciones socioculturalmente determinados, que en general se traducen en responsabilidades", que por un lado, les hace parte de la estructura familiar y comunitaria $\mathrm{y}$, por otro, les prepara para la vida adulta. En este sentido, el trabajo constituye en las comunidades indígenas, de acuerdo con Del Popolo et al (2009:38-39), un “mecanismo ancestral de socialización [en el cual] los jóvenes tienen que trabajar desde una edad muy corta, ayudando a sus padres en los quehaceres del hogar, los cultivos, el cuidado de los animales, la pesca u otro tipo de actividades que dan sustento a la familia”. 
Por lo general, la sociedad mestiza y urbana crítica a las sociedades indígenas porque en ellas, las niñas y niños participan de las actividades económicas desde temprana edad. Sin embargo, los mercados de trabajo aprovechan las capacidades y habilidades adquiridas en este tipo de socialización.

Por ejemplo, en las industrias agrícolas de México y Estados Unidos, como lo muestran Ulises Revilla y Celso Ortiz (2013: 76) los conocimientos y formas de trabajo comunitario de los hombres indígenas campesinos son explotados en el jornalerismo agrícola. Los migrantes indígenas se incorporan en los puestos de la cadena productiva dentro de empresas agrícolas como mano de obra barata, realizando "las tareas de plantado, desyerbe, instalación de estacas y estacones y fumigación", siendo las menos remuneradas y las de más riesgo. A esta situación se suma que, de manera velada o directamente, se les incorpora a los migrantes indígenas aun siendo niños. Cabe señalar que, según los datos de la Encuesta de Jornaleros Agrícolas 2009 (citada en SÁNCHEZ, 2015:79) entre la población indígena dedicada a estas labores el " $60 \%$ son niños, niñas y adolescentes que trabajan en las zonas agrícolas; $10 \%$ tiene un oficio y $30 \%$ se dedica al servicio doméstico".

Así mismo, en la ciudad, las habilidades que los varones adquieren en la vida en el campo, entre ellas, que implica fortaleza física y extenuantes horas de trabajo, les abre las puertas de la industria de la construcción, la jardinería y múltiples servicios de mantenimiento; aprendizajes que, en parte, fueron adquiriendo en lo que denominamos como trabajo comunitario, los cuales se han convertido en un capital económico en su constante migración a centros urbanos en la búsqueda de ingresos para cumplir con su compromiso fundamental como proveedores. Nos explica Patricia Arias (2009), que estas capacidades y habilidades, son por ahora, lo que les permite a los varones incorporarse al mercado asalariado en el campo y en la ciudad, pues prácticamente son la única herencia que reciben en su proceso de socialización como varones en el medio rural, ante la falta de tierras que heredar, así como de la anulación de los créditos y apoyos gubernamentales para el mantenimiento de las actividades agrícolas que decayeron a finales del siglo pasado $\mathrm{y}$ que fueron en otros momentos históricos, el eje central de la construcción de masculinidad en los medios rurales.

Por su parte, las mujeres con hijos en edad de dependencia, se mantiene dentro de los mercados locales en sus comunidades, aceptando empleos de muy baja remuneración, pero que les permite 
combinar las actividades reproductivas y productivas (ARIAS, 2009).

La socialización de las mujeres rurales, incluidas las indígenas, se desarrolla básicamente en el ámbito doméstico, y su fin es convertirlas en buenas esposas, cuidadoras y expertas en los trabajos domésticos; estas actividades son precisamente las labores que pueden ocupar las jóvenes solteras y mujeres adultas en el mercado de trabajo urbano, en el momento en que ha sido necesario que también migren del campo a la ciudad.

Ha de señalarse que hay innumerables casos en los que en estos trabajos se incorporan menores de edad, incluso niñas para llevar lo que se denomina trabajo doméstico en hogares de terceros (THAIS, 2005). Señalar también que, en el jornalerismo agrícola se adjudica a las mujeres estas mismas labores de reproducción, ya sea como una actividad remunerada o para el cuidado propio, ya que los varones se incorporan a estas actividades en compañía de sus cónyuges y sus hijas/os, para que las primeras se encarguen de los trabajos domésticos para los otros trabajadores agrícolas y los de su familia, mientras los segundos contribuyen como mano de obra como jornaleros, lo cual se organiza para incrementar entre todos los bajos ingresos que se obtienen en estas actividades para la sobrevivencia de la familia. Al respecto, la Unicef señala:

\begin{abstract}
"En México, los jornaleros agrícolas son trabajadores temporales del campo que se encargan de la siembra, la cosecha, la recolección y la preparación de productos del campo. Debido al desigual desarrollo del país, muchos trabajadores de las zonas rurales emigran a los lugares donde hay trabajo y, en muchos casos, lo hacen acompañados de sus familias. Los flujos migratorios por el trabajo agrícola son variables y afectan a todo el país.
\end{abstract}

Los hijos e hijas de los jornaleros agrícolas son un grupo especialmente vulnerable. Un $44 \%$ de los hogares de jornaleros agrícolas contaban con al menos un niño o niña trabajador, y sus ingresos se acercaban al $41 \%$ de los del total familiar.

Además, los datos arrojan que un $44.9 \%$ de las familias jornaleras en las que está presente el trabajo infantil son indígenas, de manera que la migración supone para ellos un cambio radical en sus costumbres, cultura e idioma (www.unicef.org/mexico/spanish/1704 4_17516.htm).

En años recientes en los medios rurales, las clases menos desfavorecidas o en casos de familias y comunidades que reciben remesas de migrantes internos e internacionales, ha sido posible que jóvenes, hombres y mujeres, accedan a la educación tanto en las entidades y regiones del país donde se ha introducido infraestructura educativa, o en su caso han migrado a las principales ciudades del país teniendo como objetivo el estudiar para 
completar la educación básica, media superior, superior y posgrados. No obstante, estos jóvenes con más estudios no tienen garantizada su incorporación laboral como profesionistas dentro $y$ fuera de sus comunidades como lo mostraré más adelante.

Por lo hasta ahora dicho podemos ubicar dos tipos de trabajo presentes en las comunidades indígenas rurales, el primero es el trabajo comunitario, el cual hoy en día presenta quiebres importantes por las transformaciones socioeconómicas que se han dado en el ámbito global, nacional y comunitario; cediendo su lugar, cada vez más, a una segunda forma de trabajo, el trabajo asalariado (PACHECO, 2010).

Hasta ahora, el trabajo comunitario se ha considerado por excelencia, la forma de trabajo indígena, debido a la asociación que se hace entre lo étnico y lo rural. Tal visión proviene de la política de campesinización y agriculturización de la economía rural durante las décadas de 1920-1960, en la que "las comunidades indígenas fueron integradas dentro de formas de trabajo comunales que requerían de la mano de obra de todos los integrantes de la familia, quienes, sin embargo, tenían una distribución asimétrica y desigual de las labores" (ARIAS, 2009: 72).

El resultado de este proceso es la conformación de la comunidad -como una forma de familias ampliadas $y$ emparentadas a través del matrimonio y el parentesco ritual-, estructuradas a través de la dotación de parcelas ejidales y de la actividad agrícola, en la que

"se consolidó $[\ldots]$ una economía
campesina basada en la producción
doméstica plenamente integrada al
mercado nacional a través de la venta de
productos agrícolas, pecuarios y
artesanales" (ARIZPE, 1980: 15 en
Arias, 2009: 74).

La campesinización favoreció “el arraigo de la gente en sus comunidades" a partir de la agriculturización, es decir, "de la intensificación de las prácticas agrícolas" (ARIAS, 2009:74). En este contexto el trabajo comunitario otorgó a los integrantes de la comunidad: identidad, lazos comunitarios, sentido de la vida humana a través de construcciones simbólicas de grupo, distribución de recursos, responsabilidades, deberes, obligaciones y derechos, así como formas particulares de distribución de conocimiento basadas en una organización de género y de edad rígidas. A partir de estos elementos, "las nuevas generaciones se integraban
socialmente, aunque de manera
diferenciada, de acuerdo con el sexo,
comenzando por colaborar en el trabajo
colectivo en el interior de la familia de
origen, en la que iniciaban su
socialización para participar después 
como adultos en la comunidad" (PACHECO, 2010:144).

Pese a que esta forma de vida campesina es la más extendida y ha tenido consecuencias ideológicas a largo plazo, lo cierto es que la necesidad de ingresos monetarios ha estado presente en la economía campesina, aún en su etapa campesinista y agriculturizada.

Ante la crisis del campo, a partir de la década de 1960 y, de manera generalizada, hacia la década de 1980, la necesidad de conseguir dinero en efectivo se ha incrementado, ya no sólo como un complemento, ahora se necesita para cubrir los crecientes gastos de alimentación, vivienda, salud y educación de los hijos, los cuales han recaído principalmente sobre las mujeres y los jóvenes, debido a "la disposición negativa de los hombres adultos a asumir estos gastos sin los recursos que provengan de la actividad agrícola, eje rector de su identidad de género masculina en los ámbitos rurales" (ARIAS, 2009:82). Esto último se refleja en los datos proporcionados por Mercedes Pedrero (2002:143), en su análisis basado en la Encuesta Nacional de Empleo en Zonas Indígenas (ENEZI), 1997.

Pedrero señala que, en las zonas indígenas dos terceras partes de la población ocupada total se dedican a las actividades agropecuarias: tres de cada cuatro hombres trabajan en las labores del campo; entre las mujeres la proporción es menor, pero casi llega a ser la mitad (47.4 por ciento). En números absolutos estamos hablando de 991430 trabajadores cuya actividad principal en la semana de referencia en el segundo trimestre de 1997 era la agropecuaria, de los cuales 774891 eran hombres y 216539 mujeres.

Las dos formas de trabajo comunitario y asalariado- han coexistido a través del tiempo, pero el valor, la importancia y la relación entre ambos tipos se han modificado de acuerdo al contexto político-económico, al igual que su distribución entre los sexos, las edades (ARIAS, 2009) y debemos mencionar el grupo étnico y la clase social. De tal suerte que, las mujeres con hijos/as en edades de dependencia, los hombres adultos propietarios de tierras y los adultos mayores intentan reproducir el trabajo comunitario en los ámbitos rurales, mientras las mujeres y hombres jóvenes, así como hombres adultos sin tierra o acceso a créditos van conformando flujos migratorios para incorporarse en los mercados de trabajo asalariado locales, regionales, internacionales y urbanos, donde ser hombre o mujer, mestizo o indígena, niño, joven, adulto o viejo, pobre o con recursos, de una etnia o de otra, es más, de una 
comunidad o de otra; son condiciones sociales que juegan un papel muy importante en la incorporación a los mercados laborales rurales, urbanos, de migración interna y más allá de las fronteras; estos flujos, no obstante, se ondean de acuerdo a los acontecimientos históricos, las políticas económicas y políticas, nacionales y globales.

Lo cierto es que, en el contexto referido, ante la necesidad de dinero en efectivo, la migración se convirtió en el cambio de siglo y milenio en parte de las "labores del grupo doméstico a lo largo de su ciclo" (ARIZPE, 1980: 20 en ARIAS, 2009: 83) en el que sus integrantes según su posición de género y edad participan en el fenómeno migratorio: quién se queda, quién se va, quién es un eslabón en la red migratoria, quién abre nuevos flujos, son decisiones que van dando un cariz a las formas de migrar entre los pueblos indígenas.

En este nuevo contexto, la fuerza de trabajo de las y los jóvenes ha dejado de estar integrada únicamente a los procesos de socialización de las nuevas generaciones en la vida familiar y comunitaria y considerada como una apoyo a la economía de sus grupos domésticos, ya sea en el trabajo comunitario o en el asalariado, para ser ahora este último, la pieza clave para financiar las actividades agrícolas y hacer frente a las nuevas necesidades que se fueron adquiriendo a partir de la introducción de servicios en el ámbito rural: electricidad, riego, transporte, educación y salud.

El trabajo asalariado ha servido para cubrir nuevas necesidades de consumo, impulsadas en el ir y venir de las y los migrantes, con las cuales se simboliza su incorporación a la vida del mercado capitalista.

Actualmente, el mercado laboral asalariado está en lugares cada vez más distantes al desquebrajarse las economías locales campesinas, convirtiéndose la migración en parte de los proyectos de vida de la mayoría de los jóvenes como la vía para su sobrevivencia y reproducción.

Frente a los cambios reportados en las sociedades rurales como "resultado de la acumulación de transiciones no sólo económicas, sino también demográficas, familiares y culturales que no fueron entendidas ni resueltas por el Estado ni por el mercado" (ARIAS, 2009: 261), los jóvenes indígenas y no indígenas rurales no encuentran el espacio, ni los medios para reproducir sus culturas, encontrando una salida en la incorporación al mercado de trabajo asalariado. Esta incorporación ha sido distinta para mujeres y varones.

Si bien el indicador para convertirse 
en un hombre adulto para los varones indígenas rurales había sido la incorporación total al trabajo y formar una familia de destino, y para las mujeres consistía en la capacidad de procrear; la degradación del trabajo comunitario y la consolidación del trabajo asalariado en la economía indígena, ha hecho de la migración un nuevo espacio de conformación de identidad para las y los jóvenes indígenas, tanto en su condición juvenil como étnica, ya que los ingresos monetarios les está dando la posibilidad de subsistencia, que por ahora es difícil encontrar en sus comunidades, y fuera de ellas les permite experimentar nuevas vivencias (LÓPEZ Y GARCÍA, 2016), aunque no siempre en beneficio de sus comunidades y de sí mismos, en el sentido en que su incorporación al trabajo asalariado, su participación en la economía mercantil

"no ha legitimado su participación dentro de la sociedad mestiza, ya que ésta, al estar fundada en valores mestizofílicos, desvalora la presencia de los jóvenes indígenas, a los cuales discrimina, margina y aísla de las oportunidades de educación y trabajo y los devuelve a la montaña cuando los considera excedentes" (PACHECO, 2010:152).

Para cerrar este apartado, me queda mencionar que, en las comunidades indígenas -rurales y urbanas- existen diferentes formas de trabajo que implican esfuerzos individuales y colectivos que permiten la sobrevivencia social y cultural de las familias, comunidades y pueblos, en los que las personas que son consideradas como jóvenes tienen una participación fundamental, pero en la que su condición de edad muestra particularidades expresadas en una heterogeneidad en razón del género y la clase social como intentaremos mostrar en el último apartado.

\section{Los derechos laborales de las y los jóvenes indígenas}

Junto a los derechos a la alimentación, salud, educación, protección social, vivienda y a un entorno saludable y limpio, el derecho humano al trabajo es uno de los Derechos Sociales, Económicos y Culturales.

El derecho al trabajo contiene a los derechos laborales, a saber: la oportunidad de ganarse la vida mediante un trabajo digno, libremente escogido o aceptado, salario igual por trabajo igual, condiciones dignas de trabajo, seguridad social, limitación razonable de horas de trabajo, descanso, días festivos y vacaciones periódicas pagadas, derecho a la asociación, libertad sindical y a huelga.

Para el caso de las personas indígenas 
estos derechos laborales son los mismos, pero se añaden algunas especificidades que básicamente se enuncian en tres cartas internacionales de derechos:

i. Pacto Internacional de Derechos Económicos, Sociales y Culturales

ii. Declaración de los Derechos de los Pueblos Indígenas

iii. Convenio 169 de la Organización Internacional del Trabajo

En estas cartas se reconocen para las personas indígenas los mismos derechos establecidos en los derechos laborales en las escalas internacional y nacional, pero se hace hincapié a no ser sometidas a condiciones discriminatorias de trabajo y a reconocer lo que denominé más atrás como "trabajo comunitario", en la tónica de que éste reúne características a partir de las cuales se ha conformado parte de la identidad y cultura de los pueblos indígenas.

El artículo 20 de la Declaración estipula que las personas indígenas tienen derecho, "a disfrutar de forma segura de sus propios medios de subsistencia y desarrollo, y a dedicarse libremente a todas sus actividades económicas tradicionales y de otro tipo", y cuando estos medios de subsistencia y desarrollo les han sido desposeídos, tienen derecho "a una reparación justa y equitativa" (Declaración de los Derechos de los Pueblos Indígenas,
2007, artículo 20).

En el reconocimiento de la desventaja que históricamente tienen las personas indígenas, ellas tienen el derecho a "la capacitación y el readiestramiento profesionales" para mejorar sus condiciones económicas y sociales, donde los Estados tienen la obligación de adoptar "medidas eficaces" y "especiales" para el mejoramiento de dichas condiciones (Declaración de los Derechos de los Pueblos Indígenas, 2007, artículo 21).

Sin duda el Convenio 169 sobre Pueblos Indígenas y Tribales en Países Independientes de la OIT (1979) es el instrumento internacional de mayor impacto en la transformación de la posición política de los pueblos indígenas en el mundo y en México. Este Convenio reconoce los derechos colectivos de los pueblos, sin detrimento de los derechos humanos de sus integrantes. Al igual que el Protocolo y de la Declaración, este instrumento puntualiza sobre la no discriminación de los trabajadores que pertenecen a los pueblos, aún en su calidad de migrantes, quienes deben poder ejercer los derechos laborales enunciados antes.

El Convenio 169 ofrece un papel activo a los pueblos, al reconocer el derecho que éstos tienen de "participar en la formulación, aplicación y evaluación de los planes y programas de desarrollo nacional y 
regional susceptibles de afectarles directamente" (Convenio 169, 1979, artículo 7). Otro punto importante a relevar es que se pide que los Estados adopten medidas para dar a conocer sus derechos con pertinencia cultural a quienes pertenecen a los pueblos.

En el caso de la legislación nacional, a partir de la reforma constitucional del 10 de junio de 2011 se integra lo estipulado en las cartas de derechos antes mencionados para garantizar los derechos laborales de los pueblos indígenas en los artículos: $1^{\circ}, 2^{\circ}, 5^{\circ} \mathrm{y}$ 123. En la Ley Federal del Trabajo, la reforma del 30 de noviembre de 2012 alude a las condiciones de los trabajadores indígenas en los artículos: $2^{\circ}, 3^{\circ}, 28 \mathrm{~B}, 56,133,333,336$ y 998 (COMISIÓN NACIONAL DE DERECHOS HUMANOS, 2015).

Frente a este conjunto de derechos, las personas indígenas se enfrentan a una realidad de discriminación sistemática, condiciones desfavorables y limitadas oportunidades para su desarrollo tanto de manera individual como colectiva (HORBATH, 2008), pero ¿qué significa para las y los jóvenes indígenas atravesar por estas situaciones? ¿cómo les afecta su posición como jóvenes y como indígenas en su incorporación al trabajo -comunitario y asalariado-? ¿existen estrategias de las propias comunidades y de las juventudes indígenas para lograr un acceso al trabajo de una forma acorde con los derechos labores enunciados más arriba?

Cada uno de estos cuestionamientos implicaría llevar a cabo una investigación sobre cómo opera en el caso de las y los jóvenes la discriminación, las condiciones desfavorables y las limitadas oportunidades en su incorporación al trabajo porque en razón de su edad -cronológica y social-, se les sitúa en una posición de subordinación en los diferentes sistemas sociales.

La edad es un componente de gran importancia en el análisis de la relación de los jóvenes y el trabajo. Como mencionamos en el primer apartado, en las comunidades indígenas, lo que hemos llamado trabajo comunitario ha sido un espacio de socialización de sus integrantes desde edades tempranas, lo que ha llevado a plantear una crítica desde fuera, al considerar que existe la posibilidad del trabajo infantil en la organización económica indígena. Frente a esta situación debemos considerar la enorme diferencia que hay entre integrar a las niñas, niños y menores de edad en las actividades de reproducción y producción social, y lo que consideramos como trabajo infantil, definido grosso modo como las actividades que realizan personas entre 5 y 17 años no permitidas por la ley, o que ponen en riesgo la salud o bien afectan el desarrollo físico y/o mental, o actividades de producción de bienes y servicios para el consumo de los miembros 
del hogar que afectan la salud o integridad física de quienes las realizan o se llevan a cabo en condiciones peligrosas o en horarios prolongados (INEGI, 2017).

Sin proporcionar datos precisos sobre la diferencia étnica, según los resultados del Módulo de Trabajo Infantil 2017 (MTI 2017) levantado en el cuarto trimestre en el anexo de la Encuesta Nacional de Ocupación y Empleo (ENOE), a nivel nacional "la población infantil de 5 a 17 años ascendió a 29.3 millones de personas, de las cuales 3.2 millones realizaron trabajo infantil, cifra equivalente al $11 \%$ de la población de 5 a 17 años, de los cuales eran niños el $13.6 \%$ y niñas, el $8.4 \%$. La tasa de trabajo infantil en las áreas más urbanizadas (localidades de 100 mil y más habitantes), fue del $7.6 \%$, mientras que en las menos urbanizadas (localidades menores de 100 mil habitantes) ascendió a 13.6\%.

Aún tenemos la tarea de actualizar los datos para el caso de población indígena, pero de acuerdo a la información proporcionada por Mercedes Pedrero (2002: 130), en 1997, el $16 \%$ de los niños varones entre 6 y 11 años realizaban actividades económicas, mientras que la mitad de niños de 12 y 14 años trabajaban. Con respecto a las niñas, el 9.6 entre 6 y 11 años se dedicaba a alguna actividad económica, es decir de cada 10 niñas, una ya estaba contribuyendo a la economía familiar. De las niñas entre los 12 y los 14 años su proporción era de 21.6, es decir
1 de cada 5 desempeñaba alguna actividad económica.

Con respecto a estas cifras y a lo que veníamos discutiendo sobre la diferencia que debemos considerar entre la socialización de niñas, niños y adolescentes indígenas a través del trabajo y lo que hemos definido como trabajo infantil, parece adecuado citar a la misma Mercedes Pedrero (2002:132), quien observa lo siguiente:

"La incorporación temprana al trabajo implica tanto aspectos positivos como negativos, todo depende de la forma e intensidad con que se desarrolle. Es casi imposible que una persona que no haya nacido en el campo se dedique a actividades agropecuarias; los conocimientos y valores adquiridos en el seno de la unidad productiva son difíciles de obtener en la escuela. Por el contrario, la transición hacia otros sectores es más fácil. En este sentido, el trabajo infantil en el campo es el semillero, para que sigan existiendo trabajadores agropecuarios; es decir, el trabajo infantil sólo se justifica como una estrategia de formación y capacitación. Sin embargo, cuando las tareas implican largas jornadas de trabajo a costa de su asistencia escolar y un fuerte desgaste, no sólo es un problema de justicia social para el menor, significa que su vida la inicia con desventajas que difícilmente podrá superar, justo en un mundo que cada día demanda mayor capacidad de adaptación y aprendizaje ante la diversificación de las actividades económicas y los cambios tecnológicos". 
Con la finalidad de proteger los derechos laborales de niñas, niños $\mathrm{y}$ adolescentes indígenas, los mismos instrumentos mencionados más atrás hacen alusión a protegerles del trabajo infantil, donde en la Declaración en el punto 2, dice:

\begin{abstract}
"Los Estados, en consulta y cooperación con los pueblos indígenas, deberán tomar medidas específicas para proteger a los niños indígenas contra la explotación económica y contra todo trabajo que pueda resultar peligroso o interferir en la educación de los niños, o que pueda ser perjudicial para la salud o el desarrollo físico, mental, espiritual, moral o social de los niños, teniendo en cuenta su especial vulnerabilidad y la importancia de la educación para empoderarlos" (Declaración de los Derechos de los Pueblos Indígenas, 2007).
\end{abstract}

Por su parte en el Convenio 169 de la Organización Internacional del Trabajo, el artículo 23 dispone que:

"La artesanía, las industrias rurales y comunitarias y las actividades tradicionales y relacionadas con la economía de subsistencia de los pueblos [indígenas] [...] deberán reconocerse como factores importantes del mantenimiento de su cultura y de su autosuficiencia y desarrollo económicos. Con la participación de esos pueblos, y siempre que haya lugar, los gobiernos deberán velar por que se fortalezcan y fomenten dichas actividades" (Convenio 169, OIT).
En este sentido se vuelve crucial, comprender que las sociedades indígenas si bien tienen la obligación de proteger los derechos laborales de niñas, niños, adolescentes en tanto menores de edad dentro de sus formas de organización y reproducción social, tienen también el derecho de fortalecer las formas y los espacios que permitan integrarles como parte de las familias y comunidades, y como beneficiarios del trabajo colectivo $y$ comunitario. No obstante, el Estado y la sociedad mayor tienen la obligación de crear las condiciones para que todo esto sea posible, protegiendo a las niñas, niños, adolescentes y jóvenes de excesos que lleven a la violación de derechos en sus propios grupos culturales y sociales, paralelamente que debe mantener una vigilancia acuciosa hacia la sociedad no indígena, en la cual sus integrantes desde prejuicios racistas, sexistas $\mathrm{y}$ adultocéntricos pueden llegar a ser los explotadores más crueles del trabajo de las personas más jóvenes indígenas.

\section{Formas en la que se insertan las y los jóvenes indígenas en los mercados laborales que les ofrece la ciudad}

Como lo apunté al inicio del presente artículo, las mujeres de diferentes edades y 
especialmente los jóvenes -mujeres y varones- son los grupos protagonistas de la migración indígena actual a las ciudades, pues éstas les ofrecen un mercado laboral al que pueden incorporarse, aunque la mayoría de los casos con una muy baja remuneración y sin condiciones laborales apropiadas, inicialmente por ser considerados como indígenas.

La inserción de las personas indígenas en los mercados laborales urbanos cobra diferentes matices a partir de las concatenaciones que el marcador étnico tiene con el resto de ordenadores y jerarquías sociales, conformando el complejo de la identidad para cada individuo y con respecto a los diferentes grupos y espacios de interacción de los que forman parte, es decir, de su posición en el entramado de relaciones de poder, todo lo cual configura a su vez, historias de vida, de migración y trayectorias laborales específicas que tienden a difuminarse en el análisis más general de lo que se considera como experiencia indígena en/de la ciudad.

En este apartado me propongo mostrar los matices que se presentan en la experiencia laboral de las y los jóvenes en la ciudad, en la que si bien, la condición étnica indígena es un marcador, se presentan diferencias que hay que considerar en el análisis, sobre todo, en el impulso de políticas públicas que atiendan las necesidades laborales de los jóvenes indígenas en su inserción al mercado laboral urbano de acuerdo a la etnia, comunidad y tipo de familia de procedencia, pues cada una dota de recursos sociales, económicos, culturales y habilidades laborales a sus integrantes jóvenes. Influye también la historia migratoria propia, la familiar $\mathrm{y}$ comunitaria, el tipo y características de la ciudad a la que se llega y, por supuesto, la posición en la organización de género y clase tanto en el lugar de salida como en el que deberá o podrá ubicarse en el lugar de llegada.

Particularmente, la incorporación de las y los jóvenes en los mercados laborales urbanos responde, por un lado, a las condiciones pauperizadas en los ámbitos rurales donde las familias y las comunidades ya no tienen los medios y los recursos para ofrecer un presente y porvenir a las nuevas generaciones. Por otra parte, algunas familias y comunidades han podido brindar oportunidades a sus jóvenes para que vayan a la ciudad a estudiar y, con ello, puedan escalar en la jerarquía social y tener mejores condiciones de vida, en algunos casos considerando que la formación escolar de estos jóvenes sea de utilidad para impulsar el desarrollo de sus comunidades de origen. Tal es el caso de la comunidad de Tlahuitoltepec Mixe, localizada en la sierra norte del estado Oaxaca, México. Como lo 
explica Telmo Jiménez, un joven antropólogo oriundo de esta localidad

"Esta comunidad se ha caracterizado en la región por la lucha histórica en la defensa de su lengua, territorio, formas de organización, así como la generación de propuestas educativas que desde los años setenta se ha reflejado en proyectos desde el nivel básico hasta el nivel superior, retomando elementos culturales $\mathrm{y}$ lingüísticos de la comunidad, lo que ha llevado a una resignificación étnica, y apropiación etnopolítica de la escuela. Este proceso ha sido un trabajo de varias décadas, y ha dado como resultado profesionistas en diferentes áreas, que ahora inciden en la vida comunal, en la cual las tensiones se hacen evidentes por las múltiples maneras de concebir el ejercicio de la pertenencia étnica" (Jiménez, 2018: 52).

Consecuente con el objetivo de este trabajo es momento de reflexionar sobre cómo las situaciones descritas interpelan a las y los jóvenes indígenas en el ejercicio o la violación sistemática de derechos laborales, aludiendo a tres derechos que están en tensión permanente en las experiencias de trabajo de estos jóvenes en el contexto de migración a las ciudades: a) el derecho a no tener que migrar/migrar por decisión propia y con condiciones de bienestar; b) no ser discriminados/as; y, c) contar con oportunidades; los cuales analizaremos brevemente a continuación.

El desplazamiento es una experiencia que forma parte de la historia de los pueblos indígenas, su intensificación en ciertos momentos está asociada con el despojo de sus formas de vida material y simbólica, encontrando actualmente, una situación preocupante en el sentido de que en los territorios en los que finalmente quedaron asentadas las comunidades de los distintos pueblos en el transcurso de la primera mitad del siglo $\mathrm{XX}$, han ido moviéndose $\mathrm{y}$ dispersándose como resultado del despojo de tierras, recursos y formas de vida.

Ante estas situaciones enunciadas, las comunidades han conformado diferentes estrategias para su supervivencia y reproducción; la migración de sus integrantes entre las más importantes.

Actualmente, la migración como estrategia de sobrevivencia y reproducción para las comunidades indígenas, estaría vinculada con una serie de obstáculos que, para ciertos grupos familiares, comunitarios y étnicos, les impide el poder ejercer el derecho a no tener que migrar, conformándose así la migración como un desplazamiento si no forzado, sí se ha vuelto necesario y casi la única vía para poder sobrevivir.

Ahora bien, una gran parte de las y los jóvenes que migran, aún lo hacen a través de redes familiares y de paisanazgo, pero también muchos participan en la migración motivados por la aventura de explorar y 
tener las experiencias que migrantes de retorno cuentan con gran vivacidad.

Otros jóvenes migran a partir de personas y grupos de "enganche", que llegan a las comunidades con promesas de condiciones extraordinarias de trabajo, las cuales nunca se cumplen, quedando muchas personas atrapadas en situaciones de explotación y trata laboral y sexual. Por lo que estamos hablando, no solo de un desplazamiento forzado, igualmente de incertidumbre y de peligro, donde la decisión propia y con condiciones de bienestar en el proceso migratorio difícilmente se cumplen.

Como lo es para otros migrantes, para los indígenas se han implementado muchas formas de migrar. De acuerdo con el momento y las condiciones históricas, han aparecido algunas tendencias en la forma de migrar, algunas veces combinándoles: temporal, en relevos, permanente o en diáspora; y en cada una de estas formas han participado los integrantes de familias y comunidades de acuerdo a criterios de edad, género y clase. Al respecto, Granados y Quezada (2018: 331) nos dicen que “el patrón migratorio predominante sigue siendo rural-urbano, y el perfil de los migrantes consiste en su mayoría en varones situados en las edades más productivas". Los mismos autores enfatizan esta idea al mencionar en su trabajo que "La migración indígena en el año 2015 está constituida principalmente por hombres, más que por mujeres, y el índice de masculinidad de la población indígena migrante es de 112 hombres por cada 100 mujeres" (p. 338), hemos de mencionar que la mayoría siendo jóvenes, entre los 15 y 24 años de edad.

En este sentido, las y los jóvenes como protagonistas de la migración a las ciudades, y en algunos momentos en la migración internacional obedece, por lo menos a las siguientes circunstancias.

- La densidad demográfica de los jóvenes en los contextos indígenas rurales, lo que les ha permitido conformarse como un grupo específico que demanda derechos y exige servicios, que ni las familias, comunidades e instituciones logran atender en el marco de los ámbitos rurales pauperizados.

- En los ámbitos rurales se han desarrollado en las últimas décadas, regiones agroindustriales, zonas turísticas y comerciales, que requieren de mano de obra, preferentemente con disposición a obtener baja remuneración y laborar bajo precarias condiciones.

- En las ciudades de distinto tipo, según su densidad demográfica y 
su lugar en la organización regional, nacional y global de la economía, igualmente se requiere de mano de obra para construir la infraestructura urbana que cada ciudad requiere de acuerdo a la especialización de actividades que se desarrollan en ellas, las cuales en general, ofrecen algunas oportunidades de empleo para una minoría altamente calificada y para la mayoría, un mercado laboral precarizado y segregado por sexo, edad, etnia y clase.

Cuando las y los migrantes no logran insertarse en los mercados laborales urbanos, el trabajo informal urbano se convierte en la vía para subsanar las necesidades de empleo de esta población: trabajo doméstico remunerado, comercio ambulante y servicios se convierten en nichos de trabajo para obtener ingresos monetarios.

En este contexto, la migración es un fenómeno controvertido en la vida de las y los jóvenes indígenas, pues si bien ocurre como un evento con consecuencias económicas, especialmente para quienes se mueven en búsqueda de oportunidades de trabajo cuando ya no hay opciones en el lugar de origen, también se trata de la vía que les permitirá en menor o mayor medida, "alcanzar la autoafirmación, la autonomía, la independencia económica y la participación en la toma de decisiones" en la familia y la comunidad (LÓPEZ, 2017:191). Esta situación se da en medio de imaginarios de éxito, aventura, incertidumbre y miedo, pero la búsqueda de mejores condiciones para sí y las más de las veces para su familia, les alentará a continuar con la travesía que les impone el contexto, sus propios anhelos y las huellas que van dejando quienes han migrado antes, orillándoles este conjunto de circunstancias a tener una única salida, migrar.

Lo que encuentran en los lugares de llegada, en específico en la ciudad, no es nada alentador, pues entre otras cosas se topan con la discriminación; ésta se presenta desde su llegada a la ciudad, donde desde ese momento, las personas indígenas notan la diferencia que les caracteriza como "otro", esa otredad estará presente y les causara tensión y conflicto en relación con su grupo, pero especialmente con el entorno urbano, en el cual se enfrentarán en diferentes momentos a la exclusión y los malos tratos que por su condición étnica y migrante vivirán sistemáticamente en los lugares donde habitan, trabajan, se desplazan y se divierten en la ciudad.

¿Cómo experimentan en su experiencia migratoria, la discriminación las y los jóvenes indígenas en los mercados laborales urbanos? Antes de tratar de dar 
respuesta a esta pregunta es necesario apuntar una definición sobre lo que estamos entendiendo por discriminación, para lo cual hemos de afirmar que se trata de un fenómeno que está en la base de la violación de los derechos laborales de las personas indígenas, y entre ellas los de las y los jóvenes.

Hablamos de discriminación cuando se presenta una práctica, acción, conducta o acto que impide que una persona acceda a las oportunidades o ejerza algún derecho. La discriminación puede venir de la ley, de una autoridad o persona funcionaria de instituciones públicas o privadas y de las propias personas de a pie. Cada acto discriminatorio sintetiza las diferentes condiciones sociales por las que una persona puede ser tratada como si no tuviera una condición humana, por tanto, considerada como inferior en un sentido filosófico, político y social (CONSEJO NACIONAL PARA PREVENIR LA DISCRIMINACIÓN,

www.conapred.org.mx).

La discriminación lesiona o inhabilita a quien es discriminado/a ya sea directamente, negándosele una oportunidad o derecho, aludiendo a una supuesta inferioridad con base en sus atributos y características o, indirectamente, al operar normas, reglas o prácticas que pueden ser consideradas neutras, pero que en realidad difunden un deber ser, creándose con ello estereotipos que marcan la norma frente a la diferencia y la diversidad de las personas, quienes por una serie de valores y prejuicios se considera que no encajan en un determinado estándar.

Cuando pretendemos analizar la discriminación en el ámbito económico se alude especialmente a la discriminación indirecta, pero es necesario tener en cuenta distintos elementos tanto estructurales como subjetivos, los cuales se interpelan mutuamente (MERCADO, et al, 2003). De tal suerte que, debemos considerar:

- Los entornos socioeconómicos que hacen posible el acceso a la educación, instrucción $\quad \mathrm{y}$ capacitación

- Valores familiares y de las comunidades que definen quiénes, hasta dónde y cómo sus integrantes acceden a la educación, instrucción y capacitación, por tanto, a determinadas actividades o labores.

- Calidad educacional en las escuelas y universidades.

- Posibilidad de selección del campo de especialización laboral.

- Expectativas de participación en el mercado laboral.

Desde los mercados de trabajo, la 
discriminación económica implica:

- Prejuicios de género, edad, etnia, clase, etc. de quienes contratan en los mercados laborales

- Segregación laboral con base en diferencias de género, edad, etnia, clase, etc.

- Brechas salariales basadas en la valorización y desvalorización de las actividades que realizan las personas jerarquizadas en la organización de género, edad, etnia y clase

Según datos del Banco Mundial, entrado el nuevo milenio las condiciones de vida de la población indígena en la región latinoamericana mejoró con respecto al siglo que terminó, "pero la brecha que los separa de otros latinoamericanos se ha estancado o se ha ampliado. En efecto, la pobreza afecta al $43 \%$ de los hogares indígenas de la región-más del doble de la proporción de no indígenas-y el 24\% de todos los hogares indígenas vive en condiciones de pobreza extrema, es decir 2,7 veces más frecuentemente que la proporción de hogares no indígenas". (BANCO MUNDIAL, 2015: 9). Esta situación trae consigo mayor grado de analfabetismo, menor nivel de instrucción, acceso a escuelas de más baja calidad, concentración de mano de obra poco calificada y en sectores menos remunerados, entre otras. Por otro lado, continúan muy arraigados los prejuicios sobre "lo indígena", lo cual continúa concibiéndose como símbolo de atraso, desvalorizándose las actividades en las que históricamente se ha especializado esta población, limitándoles a estar en ciertas áreas y realizar labores que se han asociado a su posición en la jerarquía social, algunas de ellas arrastradas desde la organización colonial.

Veamos cómo se manifiestan la experiencia de discriminación de acuerdo al género, la edad y la clase.

En el caso de las mujeres indígenas, por el lugar asignado al trabajo doméstico y de cuidados en relación con la idea de servilismo que se arrastra de la historia colonial (OLIVERA, 1979), las posibilidades de ocupación están limitadas a las áreas de servicio, principalmente en el empleo doméstico remunerado en sus dos modalidades, de planta y por horas, en el que la mayoría de las mujeres indígenas de todas las edades, grupos étnicos, e incluso estratos sociales se emplean por el hecho de ser mujeres y de provenir de medios rurales e indígenas en este nicho laboral (DURÍN, 2009).

De acuerdo con lo anterior, el propio campo laboral del trabajo doméstico remunerado es dinamizado por las 
características particulares de quienes se insertan en él, por ejemplo, en el llamado servicio de planta, son las jóvenes que migran solas, la mayoría solteras y $\sin$ hijas/os, el grupo que predomina en esta modalidad porque al ser recién llegadas e incorporarse por primera vez al trabajo asalariado, la remuneración que reciben, incluye un pago en especie, hospedaje y alimentación, lo cual les permite ahorrar para enviar remesas a sus familias o para comprar productos de consumo urbanos que les hace sentirse parte de la ciudad y construir una experiencia juvenil (LÓPEZ, 2017). De tal suerte que, las jóvenes en el servicio de planta predominan porque en su identidad se sintetizan condiciones sociales dadas por la etnicidad, género, edad y situación migratoria, que en conjunto les coloca en un lugar de fuerte discriminación y vulnerabilidad, restringiéndoles sus posibilidades laborales, orillándoles a emplearse en un nicho de trabajo considerado de nulo o poco prestigio $\mathrm{y}$ estigmatizado. En situaciones similares las jóvenes se insertan en el comercio, especialmente ambulante, en menor medida en fábricas y, desafortunadamente, en el trabajo sexual (TORIZ, 2014).

Ante las situaciones descritas, la vida laboral de las jóvenes indígenas que migran a la ciudad está, en general, desprovista del cumplimiento de sus derechos laborales.
Sabemos que con respecto al trabajo doméstico remunerado se ha avanzado en el reconocimiento y valorización de este trabajo, y como tal de los derechos concomitantes a éste. No obstante, para ello se requiere, en el caso de las niñas, adolescentes y jóvenes indígenas que en las legislaciones se les tenga en cuenta como un grupo específico dentro de las trabajadoras del hogar para quienes la posición de edad tiene un lugar fundamental en su condición como empleadas en este nicho laboral.

El género tiene otra connotación para el caso de los varones, si bien es un marcador que les adjudica un lugar en la segmentación laboral, es la etnicidad en concatenación con la edad y la clase, como se sintetizan las desigualdades, específicamente para los jóvenes con menores recursos.

También es el área de servicios en la que los varones indígenas mayormente logran insertarse laboralmente en la ciudad. No obstante, las posibilidades de empleo están ligadas a una serie de oficios como albañiles, carpinteros, ebanistas, jardineros, macheteros, estibadores, tablajeros; en cuyo caso pueden empezar a participar como aprendices con los varones de las redes familiares y comunitarias, o empleándose directamente con contratistas y empleadores urbanos en la ciudad. Dependiendo de las mismas redes y de las 
habilidades que cada individuo tenga y adquiera, hay posibilidad de ascender en el escalafón que ofrece cada uno de estos oficios, incluso, convertirse en contratistas, dueños de su propio negocio o taller. Aunque en razón de la etnicidad indígena y la clase, llegar al más alto nivel que ofrecen estos diferentes oficios, es prácticamente imposible para la mayoría ellos, especialmente para los jóvenes indígenas, quienes están subsumidos en razón de la etnia, la edad y la clase en las jerarquías que suponen tales oficios.

La participación de los jóvenes indígenas en estos oficios, tal como lo documenta Sánchez (2009) en su investigación con trabajadores de la construcción en la Ciudad de México, viven en mundo masculino, fuertemente estratificado por la edad, etnicidad y clase, esto último en términos de capitales y recursos disponibles. Tal estratificación se reproduce y mantiene las fronteras entre unos grupos y otros mediante la violencia cotidiana dentro de cada estrato y entre segmentos. Cabe mencionar que, entre los distintos gremios si acaso se tienen algunos derechos laborales, estos siempre son endebles, de acuerdo a sí se trabaja por cuenta propia, de manera informal o formal, en una pequeña, mediana o gran empresa.

En el pasado, los oficios mencionados trazaron la trayectoria laboral de los varones a lo largo de su vida. Las circunstancias laborales actuales impiden la permanencia por largo tiempo en los empleos que estos oficios ofrecen, la mayoría son temporales, sin contratos de por medio ni prestaciones sociales básicas como la seguridad social, aunque estos oficios suelen tener riegos a la salud e integridad física, por lo tanto, los trabajadores suelen ir de un empleo a otro, de acuerdo a las condiciones laborales que ofrecen, pero también en relación con las necesidades y la tolerancia de cada trabajador para hacer frente a la hostilidad laboral que suele vivirse en estos espacios.

Podemos decir hasta ahora, que los jóvenes varones indígenas al igual que su contraparte femenina comparten condiciones laborales precarias, ante esto no podemos afirmar de entrada que unas $u$ otros tengas mejores condiciones para ejercer sus derechos laborales en su inserción a los mercados de trabajo urbanos, pero encontramos diferentes formas de concatenación de los ordenadores sociales que atraviesan su identidad para participar en un espacio laboral segmentado, que se traduce en ingresos diferenciados para unas y otros.

En el caso del trabajo doméstico remunerado los ingresos son menores frente a los varones que se dedican a los oficios señalados. Como se mencionó en el primer caso, los ingresos pueden ser 
pagados en especie. Algunas niñas, adolescentes y jóvenes no reciben el pago por sus servicios directamente, pues se les hace llegar a sus padres o tutores, situaciones que yo misma reporté en mi investigación con jóvenes indígenas migrantes que se incorporan en este tipo de trabajos (LÓPEZ, 2017). Especialmente, los ingresos suelen ser bajos por la desvalorización que hay del trabajo doméstico asociado con las mujeres para quienes, si bien se les distribuye actividades según la edad, entre las más jóvenes, las adultas y las adultas mayores, tal como lo muestra Alejandra Toriz de la Rosa (2014), y según la etnia entre las migrantes rurales indígenas y mestizas frente a las habitantes pobres de la ciudad que también están asociadas con este tipo de trabajo. Lo cierto es, que la nula posibilidad de desarrollo, de obtener ingresos dignos y mejores condiciones de empleo son compartidas por todos los estratos que conforman este nicho laboral.

Los ingresos son más altos para los varones que se incorporan a los oficios mencionados, asociados a un perfil como varones, aquellos que están "hechos para el trabajo manual y rudo", el cual se considera, no requiere capacitación especializada, pero sí, cumplir con una trayectoria en la que se empieza como aprendiz, se escala por

\footnotetext{
${ }^{1}$ Hablantes de lengua indígena
}

distintos niveles para finalmente llegar a ser "el maestro"; cada escalafón corresponde con un ingreso que va incrementando, pero también con la posibilidad de dejar de hacer el trabajo más pesado y agotador. Pero, la oportunidad de avanzar en la jerarquía en estos oficios estará fuertemente ligada con la edad, la etnia y la clase.

Ahora bien, en las últimas décadas para la población en general, particularmente en los medios rurales, las generaciones jóvenes han podido acceder a la educación formal en los diferentes niveles, aún en el medio superior, superior y posgrados.

“En 2015 sólo 72.6\% de la población indígena y $67.5 \%$ de $\operatorname{los}$ HLI $^{1}$ de 12 a 14 años concluyeron el nivel de primaria, a diferencia del resto de la población, donde $81.7 \%$ alcanzó este nivel. La población autoadscrita como indígena se encuentra en una mejor situación que la indígena, ya que $76.7 \%$ terminó la primaria, es decir, 4 puntos porcentuales por arriba de la población indígena $[\ldots] \quad 55.8 \%$ de indígenas, $48.3 \%$ de HLI y $60.6 \%$ de la población autoadscrita contaban con los antecedentes para ingresar a $\mathrm{EMS}^{2}$, a diferencia del resto de la población, cuya proporción fue de $68.2 \%$ " (ROBLES y PÉREZ, 2017: 14-15).

En 2005, de acuerdo con los datos analizados por Gallart y Hernríquez (2006)

\footnotetext{
${ }^{2}$ Educación Media Superior
} 
solo el $4.7 \%$ de la población indígena entre 15 y 64 años logró cursar al menos un grado de educación superior, con respecto al 14 . $4 \%$ de la población no indígena.

Si tomamos en cuenta que uno de los impedimentos que tienen los jóvenes indígenas para su inserción en aquellas áreas de trabajo con mejores condiciones tiene que ver con la falta de escolarización y capacitación, nos podemos preguntar ¿qué está pasando con las y los jóvenes indígenas que sí tienen escolaridad y capacitación, incluso profesional? ¿Qué está ocurriendo con las personas indígenas en los diferentes niveles escolares, qué condiciones les permiten o les obstaculizan su inserción para avanzar en sus estudios y egresar, qué beneficios les trae la escolarización y dónde se desarrollan profesionalmente? Son preguntas que están en el aire, una vez que las organizaciones indígenas han logrado que la educación sea uno de los derechos de los pueblos indígenas reconocido por el Estado, obligándole a garantizar su ejercicio, creando infraestructura educativa en las propias zonas indígenas, así como programas que permitan la participación de la población indígena en la educación en todos sus niveles (OLIVERA y DIETZ, 2017).

Ante los cuestionamientos planteados, se advierte primero, las desventajas con las que la población indígena se incorpora a la educación, las cuales se arrastran a su vez en su inserción laboral, de tal manera que los egresados universitarios indígenas no cuentan todavía con redes necesarias para incorporarse exitosamente en los distintos mercados labores para cubrir las expectativas de ocupación de los jóvenes profesionistas (Martínez y Muller, s/f); muchos de los cuales se constituyen o se integran en asociaciones civiles, organizaciones y consultorías, que les permiten poner al servicio de sus comunidades y de la sociedad en general sus saberes universitarios (Información de campo).

En este sentido, la profesionalización de las nuevas generaciones se convierte en un capital que es apropiado en los espacios organizativos indígenas (LÓPEZ, 2018), esto a su vez les catapulta a ocupar puestos de representación popular o en funciones en diferentes instancias de gobierno, particularmente en las que atienden asuntos indígenas. Sin embargo, en general, las y los jóvenes indígenas profesionalizados cuentan con muy pocas oportunidades para incorporarse en el mundo del trabajo empresarial, académico/ científico, de educación y salud, o de las especializaciones profesionales en las que se califican. Al respecto Martínez y Muller (2006: 6-7) nos explican lo siguiente: 
"Las opciones laborales para los indígenas resultan menores a las del total de la población mexicana [...] esas diferencias no se justifican únicamente por una menor escolarización, por lo que las políticas de becas a los estudiantes no son suficientes para mejorar el nivel de vida de los hogares indígenas. La migración es cada día más una opción para la movilidad social, pero también en las ciudades medias y grandes el perfil ocupacional es segregado".

Bajo tal planteamiento, la etnicidad juega un lugar central en el contexto de inserción de estos jóvenes en el mercado laboral y, sin duda, el género marcará diferencias por el lugar que ocupan en las jerarquías entre los sexos, mujeres y hombres, donde el lugar subordinado de las primeras y los prejuicios que persisten sobre su condición social como es la negación de la educación porque se considera que en algún momento deberán casarse y no requerirán más capacitación que la que la propia familia le otorgue para ser buena esposa, siguen siendo un obstáculo para que las niñas, jóvenes y mujeres acudan a la escuela y se profesionalicen (BONFIL, 2003).

Específicamente, la etnicidad como un atributo negativo hará que algunos jóvenes profesionistas traten de borrar las marcas étnicas o solo las harán visibles cuando éstas sean un capital o requisito para avanzar en el espacio laboral o cuando exista un ambiente propicio para expresar la diferencia.

Lo cierto es que, la experiencia de las y los jóvenes indígenas como profesionistas en diferentes ámbitos sigue siendo muy específica frente al conjunto de jóvenes sin instrucción escolar o bajos niveles educativos, además de que aún es reciente; estas circunstancias no han permitido conformar una masa crítica desde la cual se puedan tender redes laborales para la incorporación de los jóvenes indígenas profesionistas, ni en sus lugares de origen y menos en la ciudad, para ello se necesitan acciones afirmativas no solo en las áreas educativas y de capacitación, es menester que las empresas e instituciones laborales establezcan cuotas de participación laboral de las y los profesionistas indígenas, y que en las propias comunidades que incentivan la educación de sus jóvenes se establezcan mecanismos de integración de sus conocimientos para beneficio colectivo, pero también individual, en el marco de la organización comunitaria.

En este apartado he intentado dar un panorama general sobre cómo las personas jóvenes indígenas se insertan en los mercados laborales urbanos, advirtiendo algunos claroscuros en las posibilidades de incorporación laboral de las y los jóvenes indígenas en su experiencia como migrantes trabajadores, pero también como 
estudiantes y profesionistas. Sé que no se agota la amplia heterogeneidad de experiencias laborales, pero sí espero haber dejado una posible forma de abordaje de estas diversas expresiones de inserción de las juventudes indígenas en los mercados laborales urbanos, en las que vemos que enfrentan la violación de los derechos laborales que han sido estipuladas en cartas de derechos internacionales y nacional. Al respecto, hay mucho por hacer desde una acción co-responsable entre las propias familias y comunidades, el Estado, la sociedad, los organismos y los propios jóvenes indígenas, quienes no podemos considerar desde ningún punto de vista como pasivos ante esta situación.

\section{Reflexiones finales}

En este artículo, puntualicé en las formas de trabajo comunitario y asalariado que han coexistido en el marco de la organización de las comunidades indígenas, en el que el segundo ha tomado cada vez mayor relevancia ante la descampesinización y desagriculturación de la economía indígena, y de la necesidad de trabajo de baja remuneración en las ciudades, donde la mano de obra indígena ha sido incorporada, especialmente en los mercados laborales de servicios e informales.

Se describieron algunas cartas de derechos, en las que se hace mención de los derechos laborales que tienen las personas consideradas indígenas. En este punto, observamos la importancia de la participación que tienen niños y jóvenes en la economía indígena, no solo en un sentido propiamente económico, sino porque ha sido la forma de socialización de estos grupos de edad en la familia y la comunidad. Esto me llevó, por supuesto, a plantear algunas reflexiones sobre el trabajo infantil, que para el caso de los indígenas es visto desde la sociedad no indígena desde un doble estándar. Es decir, es malo cuando se utiliza la mano de obra de niños y jóvenes menores de edad dentro de las comunidades indígenas, pero pasa este tema desapercibido cuando la sociedad blancomestiza hace uso de ella, justificándose en explicaciones racistas, sexistas, clasistas y adultocéntricas.

En la última parte de este documento, describí de manera muy general las posibilidades de empleo para mujeres indígenas en la ciudad, siendo el empleo doméstico remunerado la mayor fuente de ocupación para ellas. Los varones indígenas se insertan en distintos oficios, en los que la etnia limita sus posibilidades de ascenso en los escalafones que permiten los mismos. Una mención hago de los profesionistas 
indígenas, quienes pesé a ser mano de obra calificada, aún no cuentan con redes que les permitan acceder a los mercados con mejores condiciones laborales.

El trabajo doméstico remunerado constituye una actividad económica para las mujeres de distintas generaciones, pues ellas están capacitadas para realizar las tareas domésticas y existe un mercado laboral, que con altas y bajas oferta posibilidades para la obtención de ingresos para las mujeres indígenas y rurales, pero en este espacio se destacó el lugar que la edad tiene en la dinamización de este nicho laboral. Mientras que para los varones que se incorporan a diferentes oficios, la síntesis entre edad y etnicidad es el punto de quiebre donde se manifiestan las posibilidades y obstáculos para escalonar en una jerarquía laboral, marcada por relaciones de violencia y hostilidad entre los propios varones.

Un dato que sobresale de la información expuesta en este artículo apunta a que la generación joven está alcanzando niveles escolares más altos que sus antecesores, tan solo entre el censo de 2000 y el conteo intercensal 2005, si bien se mantuvo el mismo porcentaje de primaria completa 19.9 entre la población indígena de 15 a 64 años, en secundaria se incrementó de 13.1 a 17.0, preparatoria de 8.4 a 10.9 y educación superior de 4 a 4.7 , pero esto no significa mayores oportunidades laborales para la generación de los jóvenes, ya que pesa sobre éstos un sistema económico desigual basado en las diferencias de género, étnicas, edad y clase.

Para el caso de los jóvenes indígenas, la defensa del derecho al trabajo y los derechos laborales concomitantes a éste, alude, de acuerdo al contexto descrito a un conjunto de derechos que no podría pensarse directamente que están relacionados con el trabajo y las condiciones laborales, como lo es, el derecho a no tener que migrar, éste está relacionado a su vez con la defensa del territorio, la cultura y la economía indígenas. En razón de esta apreciación, el ejercicio de los derechos laborales para las juventudes indígenas va más allá de programas de formación, capacitación y participación en el sistema educativo, o de las condiciones que haya en los mercados laborales, ha de considerarse al mismo tiempo, el apoyo a proyectos productivos que involucre a las generaciones más jóvenes en los propios lugares de origen, una revisión de cómo afectan los megaproyectos las condiciones de vida y laborales de los jóvenes presentes y de las generaciones futuras. Asímismo, es importante considerar la experiencia y la condición juvenil como valiosa en sí misma, pero entendiéndola siempre en un amplio contexto de relaciones sociales de poder. 


\section{Referencias bibliográficas}

ARIAS, Patricia. "Del desarraigo a la diáspora." Dilemas de la familia rural. México, UDG/CUCSH, Cámara de Diputados, Ed. Miguel Ángel Porrúa, 2009.

COMISIÓN PARA EL DESARROLLO DE LOS PUEBLOS INDÍGENAS. Indicadores socioeconómicos de los pueblos indígenas de México, 2015. Consulta en línea: https://docplayer.es/71794171-Indicadoressocioeconomicos-de-los-pueblos-indigenasde-mexico-2015-coordinacion-general-deplaneacion-y-evaluacion.html

BANCO MUNDIAL. Latinoamérica Indígena en el Siglo XXI. Banco Internacional de Reconstrucción y Fomento, 2015.

BONFIL, Paloma. “¿Estudiar para qué? Mercados de trabajo y opciones de bienestar para las jóvenes del medio rural. La educación como desventaja acumulada". En: Enrique Pieck (coord.). Los Jóvenes y el Trabajo. La Educación frente a la Exclusión Social. Instituto de Investigaciones para el Desarrollo de la Educación. México: Universidad Iberoamericana, pp. 527-549, 2003.

COMISIÓN NACIONAL DE DERECHOS HUMANOS. El derecho humano al trabajo de las personas indígenas. México, CNDH, 2015.

DEL POPOLO, Fabiana, Mariana LÓPEZ y Mario ACUÑA. Juventud indígena y afrodescendiente en América Latina: inequidades sociodemográficas y desafíos de políticas. Chile, CEPAL, 2009.

DURÍN, Séverine. En Monterrey hay trabajo para mujeres. Procesos de inserción de las mujeres indígenas en el Área Metropolitana de Monterrey. México: UNESCO, CIESAS, CDI, 2009.

GALLART NOCETTI, María Antonieta y Cristina HENRÍQUEZ BREMER.

"Indígenas y educación superior: algunas reflexiones". Universidades [en linea] 2006, (julio-diciembre) : Disponible en: $<$ http://www.redalyc.org/articulo.oa?id=3 7303206> ISSN 0041-8935 Fecha de consulta: 18 de junio de 2019

GRANADOS ALCANTAR, José Aurelio y María Félix QUEZADA RAMÍREZ. "Tendencias de la migración interna de la población indígena en México, 19902015". Estudios Demográficos y Urbanos vol. 33, núm. 2 (98), mayo-agosto, pp. 327-363, 2018

HORBART, Jorge. "La discriminación laboral de los indígenas en los mercados urbanos de trabajo en México: revisión y balance de un fenómeno persistente". En Fernando García (ed.) Identidades, etnicidad y racismo en América Latina, Quito, Colección 50 años. FLACO, pp. 121-158, 2008.

JIMÉNEZ DÍAZ, Telmo. "Los espacios comunitarios como escenarios de disputa generacional: lo juvenil frente al sistema normativo interno en Tlahutoltepec Mixe, Oaxaca". En: Jahel López Guerrero y Marcela Meneses (coords.) Jóvenes y espacio público. México, CEIICH/IIS, pp. 51-64, 2018.

LÓPEZ GUERRERO, Jahel y Luis Fernando GARCÍA ÁLVAREZ. "La construcción de lo juvenil en las experiencias migratorias de mujeres y hombres jóvenes indígenas en dos contextos metropolitanos en México". En: Aremy Evangelista García, 
Tania Cruz Salazar y Ramón Abraham Mena Farrera (coords.) Género y Juventudes. México, Colegio de la Frontera Sur, Colección de Estudios de Género en la Frontera Sur, pp.45-77, 2016.

LÓPEZ GUERRERO, Jahel. "Mujeres jóvenes indígenas migrantes en la zona metropolitana del valle de México: condiciones estructurales y subjetividades en la construcción de su experiencia juvenil." México, CEIICH-UNAM, 2017.

LÓPEZ GUERRERO, Jahel. "La participación de las mujeres indígenas jóvenes en los espacios organizativos como vía para construir ciudadanía: el caso de México". En: Jahel López Guerrero y Marcela Meneses (coords.) Jóvenes y espacio público. México, CEIICH/IIS, p. 6586, 2018.

\section{MARTÍNEZ CASAS Regina y Lara} MULLER. "Escolarización y trabajo en los pueblos indígenas: De la segregación educativa a la discriminación laboral en las estadísticas mexicanas". Ponencia, Cocoyoc, Morelos, 2006. Recuperado de: http://www.cdi.gob.mx/sicopi/migracion_o ct2006/6_regina_martinez_casas_educacio n_y_trabajo.pdf

\section{MERCADO, Alejandro F., ANDERSEN,} Lykke E., \& MURIEL H., Beatriz. "Discriminación étnica en el sistema educativo y el mercado de trabajo de Bolivia”. En: Revista Latinoamericana de Desarrollo Económico, (1), pp. 69-98, 2003.

OLIVERA, Mercedes. "Sobre la explotación y opresión de las mujeres acasilladas en Chiapas”. En: Cuadernos Agrarios, año 4, no. 9, septiembre, pp. 43-55, 1979. OLIVERA RODRÍGUEA, Inés y Gunther DIETZ. "Educación superior y pueblos indígenas. Marcos para contextualizar". Anthopologica. Año XXXV, No. 39, pp. 739, 2017.

\section{PACHECO LADRÓN DE GUEVARA,} Lourdes. "Los últimos guardianes. Jóvenes rurales e indígenas”. En: Rossana Reguillo (coord.). Los jóvenes en México. México, FCE/CONACULTA (Biblioteca mexicana), 2010, pp.124-153. PEDRERO NIETO, Mercedes. "Empleo en zonas indígenas". En: Papeles de POBLACIÓN, CUEAP/UAEM, No.3, pp. 117-162, enero-marzo, 2002.

\section{REVILLA LÓPEZ, Ulises y Celso ORTIZ} MARÍN. "Etnificación del mercado de trabajo agrícola en California, Estados Unidos, y Sinaloa, México". En: Iztapalapa, Revista de Ciencias Sociales y

Humanidades, 75, 2013, pp. 57-81.

Recuperado de:

http://www.redalyc.org/articulo.oa?id=3934 8329004

\section{ROBLES VÁZQUEZ, Héctor y Mónica} Gladys PÉREZ MIRANDA (coords.). Breve panorama educativo de la población indígena. Día Internacional de los Pueblos Indígenas. Instituto Nacional para la Evaluación Educativa, México, 2017.

SÁNCHEZ CHÁ VEZ, José Ángel. "Jóvenes, identidades migrantes, subcultura y performance." Tesis Ingeniería en Agronomía (especialidad en Sociología Rural). Universidad Autónoma Chapingo, México, 2009.

SÁNCHEZ GARCÍA, Carolina. "La migración indígena, interna e internacional”. En: Genoveva Roldán Dávila, Carolina Sánchez García (coords.) Remesas, migración y comunidades indígenas de México. México, D.F.: Universidad Nacional Autónoma de México, Instituto de 
Investigaciones Económicas, pp. 71-90, 2015.

THAIS. Trabajo adolescente doméstico en hogares de terceros en el Distrito Federal. México, Thais Desarrollo Social, S.C./ Instituto Nacional de Desarrollo Social, 2005.

TORIZ DE LA ROSA, Alejandra. "Una mirada al trabajo remunerado en el hogar de las jóvenes indígenas en la ciudad de México". En: Maya Lorena Pérez Ruiz y Laura Raquel Valladares de la Cruz (coords) juventudes indígenas. De hip hop y protesta social en América Latina. México, Instituto de Antropología e Historia. Colección Etnología y Antropología Social. Serie Enlace, pp. 351-384, 2014

Fuentes documentales y sitios web

Convenio 169 de la Organización

Internacional del Trabajo, 1979

Consejo Nacional para Prevenir la

Discriminación, www.conapred.org.mx

Declaración de los Derechos de los Pueblos Indígenas, 2007

Módulo de Trabajo Infantil, Principales

Resultados. INEGI, 2017.

Pacto Internacional de Derechos

Económicos, Sociales y Culturales

Unicef. Jornaleros agrícolas.

www.unicef.org/mexico/spanish/17044_175 16.htm

http://redtrabajadorasdelhogar.blogspot.com

https://www.sinactraho.org.mx/ 